\title{
Wheal Mean Diameter
}

National Cancer Institute

\section{Source}

National Cancer Institute. Wheal Mean Diameter. NCI Thesaurus. Code C112428.

The mean diameter of the flat, circular, slightly raised area that forms around the site of an antigenic challenge to the skin. 